> Le secteur des dispositifs médicaux implantables est un secteur des produits de santé en pleine expansion et particulièrement dynamique dans le domaine de la recherche. En effet, pour améliorer la prise en charge des patients et s'adapter au mieux aux exigences cliniques, les chercheurs du domaine conçoivent de nouveaux types de dispositifs médicaux. Pour cela, ils utilisent différentes familles de biomatériaux présentant des caractéristiques chimiques et physiques très variées de façon à proposer aux cliniciens des produits de santé parfaitement adaptés aux applications biomédicales. Dans cet article, nous montrons, grâce à un exemple, comment à partir d'une famille de biomatériaux (les polymères dégradables), il est possible de concevoir un dispositif médical implantable pour la prise en charge thérapeutique de la rupture du ligament croisé antérieur. Les principales étapes conduisant à la conception d'un renfort ligamentaire total sont détaillées dans cette étude. Elles vont de la synthèse et la caractérisation de polymères dégradables jusqu'à la mise en forme en tricot, en passant par l'étude de l'influence de la stérilisation sur les propriétés mécaniques et la vérification de la cytocompatibilité. <

Parmi les divers domaines de l'industrie des biens de santé, le secteur du dispositif médical connaît une croissance mondiale soutenue de $6 \%$ [1]. Ce secteur comporte une très grande variété de produits de santé, des gants chirurgicaux aux tests de grossesse, en passant par des stimulateurs cardiaques ou des équipements d'imagerie médicale. Notre propos se focalisera sur une famille de dispositifs, les dispositifs médicaux implantables (DMI) dont la définition est «Tout dispositif destiné à être implanté en totalité dans le corps humain ou grâce à une intervention chirurgicale, et à demeurer en place après l'intervention ». On peut citer les prothèses en chirurgie orthopédique (prothèses de

\section{Polymères synthétiques dégradables pour la conception de dispositifs médicaux implantables}

\section{Le cas de la} ligamentoplastie

Xavier Garric, Benjamin Nottelet, Coline Pinese, Adrien Leroy, Jean Coudane

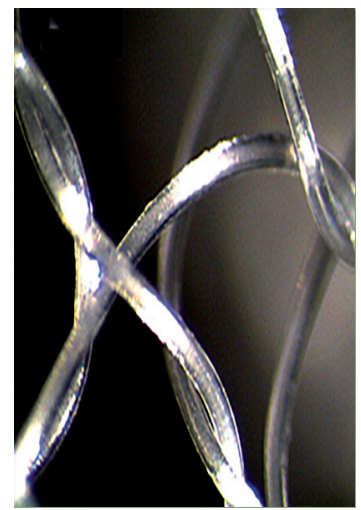

Département Biopolymères Artificiels : Institut des Biomolécules Max Mousseron (IBMM), UMR 5247, CNRS, Université Montpellier, ENSCM, Faculté de Pharmacie, Bâtiment I,

15, avenue Charles Flahault, BP14491, 34093 Montpellier Cedex 5, France. xavier.garric@umontpellier.fr

hanche, de genou, de coude, etc.), en chirurgie cardio-vasculaire (stents, valves cardiaques, etc.), en chirurgie viscérale ou gynécologique (treillis pour hernie abdominale ou prolapsus génital). Tous ces DMI sont conçus à partir de biomatériaux. Les Conférences de Chester de la Société Européenne des Biomatériaux ( $(S B)$, dites conférences du consensus, ont, en 1986 puis en 1991, retenu la définition suivante du biomatériau: "matériau non vivant, utilisé dans un dispositif médical et conçu pour interagir avec des systèmes biologiques, qu'il participe à la constitution d'un appareillage à visée diagnostique ou à celle d'un substitut de tissu ou d'organe, ou encore à celle d'un dispositif de suppléance (ou assistance) fonctionnelle».

Afin de répondre parfaitement aux exigences du cahier des charges d'une application clinique, ces biomatériaux doivent présenter des propriétés et des caractéristiques très précises. En premier lieu, le biomatériau doit être biocompatible: il ne doit pas provoquer d'infections, ne doit pas contenir de substances toxiques et/ou cancérigènes, et ne doit pas provoquer de réaction inflammatoire ni de rejet. Ensuite, les propriétés mécaniques du dispositif médical doivent être en adéquation avec celles des tissus hôtes et avec les contraintes mécaniques qui sont relatives au processus de cicatrisation. Enfin, les biomatériaux doivent être compatibles avec les contraintes physiques et chimiques lors de leur utilisation dans l'organisme mais également 


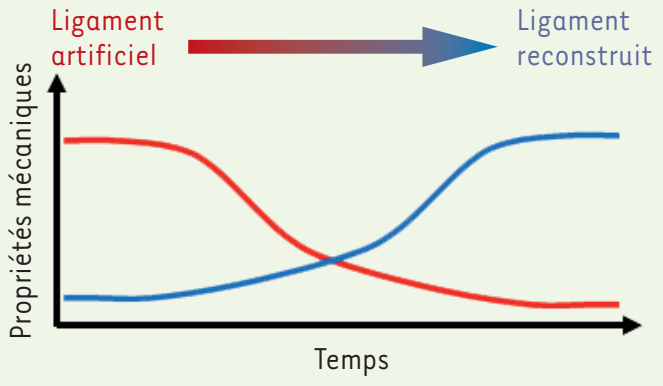

Figure 1. Représentation « idéale 》 des variations des propriétés mécaniques du ligament artificiel et du ligament en reconstruction.

lors du procédé de fabrication comme de la stérilisation, obligatoire avant toute implantation dans un organisme.

Parmi les divers types de biomatériaux (métaux et alliages, céramiques, polymères), les polymères prennent une place de plus en plus importante du fait de leur grande variété de compositions et donc de propriétés, et de leurs applications extrêmement nombreuses. Ils peuvent être d'origine naturelle ou synthétique, dégradables ou non pour des applications temporaires ou permanentes. Tous les domaines de la médecine et de la chirurgie sont concernés (endocrinologie, urologie, cardiologie, orthopédie, stomatologie, ophtalmologie, etc.). Pour illustrer l'utilisation des polymères synthétiques dégradables pour la conception de dispositifs médicaux implantables, nous nous appuierons sur des exemples de biomatériaux susceptibles de remplacer le ligament croisé antérieur du genou (LCA), un défi actuel très important en chirurgie orthopédique.

\section{Polymères dégradables et reconstruction ligamentaire}

Lors de la reconstruction du ligament croisé antérieur du genou (LCA), les greffes autologues sont utilisées par les chirurgiens pour fournir au genou des fibres de collagène, un polymère naturel résistant aux contraintes physiologiques et permettant la réparation tissulaire. Bien qu'étant actuellement la technique la plus satisfaisante pour les chirurgiens, elle présente néanmoins des inconvénients, notamment le temps de préparation de la greffe qui représente plus de la moitié du temps d'opération, et la morbidité due au prélèvement de tissus sains chez le patient. Les solutions thérapeutiques se sont progressivement orientées vers l'utilisation de biomatériaux exogènes tels que des fibres de carbone, de poly (éthylène téréphtalate) (PET), de poly(tétrafluoroéthylène) (PTFE) ou de polypropylène (PP) [2]. Cependant ces composés non dégradables ont montré leurs limites: des phénomènes d'abrasion entraînent en effet la rupture du ligament à long terme et la recolonisation par les tissus ne se réalise pas de manière optimale [3]. Idéalement, les cellules doivent coloniser la prothèse et produire un néo-tissu qui prend progressivement le relais de la prothèse qui assure la stabilité du genou en attendant d'être remplacée par le nouveau ligament. Ce néo-tissu ne pourra être efficace que s'il est soumis à une contrainte [4]. Il est donc nécessaire que la prothèse perde progressivement ses propriétés mécaniques et que, de manière concomitante, le néoligament gagne en propriétés mécaniques pour que l'ensemble puisse assurer la stabilité du genou de façon continue (Figure 1) [5].

La réponse à cette problématique ne peut être apportée que par l'utilisation de biomatériaux dégradables. Ceux-ci peuvent être d'origine naturelle, mais ils souffrent d'un manque de propriétés mécaniques (c'est le cas du collagène) ou d'une dégradation trop lente dans l'organisme (comme la soie). Les recherches se sont donc orientées vers des polymères de synthèse. Ces biomatériaux doivent être biocompatibles, y compris durant leur période de dégradation, et leur utilisation autorisée par la FDA (Food and drug administration) aux États-Unis, et par les instances européennes. Notre choix s'est donc porté sur les dérivés de l'acide polylactique qui répondent à ces critères.

Un cahier des charges regroupant les caractéristiques essentielles du renfort ligamentaire a ensuite été élaboré. Il se base en premier lieu sur les propriétés mécaniques du ligament croisé qui supporte des contraintes importantes au niveau du genou. Le LCA, chez un homme, possède une résistance et une contrainte à la rupture comprises respectivement entre 1100 et 2500 Newton ( $\mathrm{N}$ ) et entre 13,7 et 36,4 MPa (mégapascal) [5]. En sollicitation cyclique, fréquente dans le genou, le ligament se déforme sous la contrainte et revient dans son état de tension initial. Sa rigidité est notamment reflétée par la valeur du module d'young ${ }^{1}$, comprise entre $50 \mathrm{MPa}$ et $150 \mathrm{MPa}$, et sa raideur, comprise entre 111 et $397 \mathrm{~N} / \mathrm{mm}$ [5].

Comme pour tous les matériaux destinés à être utilisés dans un organisme vivant, d'autres critères doivent être appréhendés lors de la préparation d'un LCA artificiel. II doit avoir une vitesse de dégradation compatible avec la vitesse de reconstruction du tissu. Selon la littérature [6], ce temps de reconstruction est compris entre trois mois, pour obtenir une structuration collagénique mécaniquement satisfaisante, et deux ans pour une cicatrisation complète avec une structure tissulaire identique au tissu natif. Le matériau utilisé doit ainsi fournir une assistance mécanique temporaire pendant les trois premiers mois puis se dégrader progressivement pour laisser la place au néo-tissu. II doit aussi pouvoir prendre la forme de fil/tresse/filament/treillis afin de fournir une structure cylindrique et poreuse qui sera compatible avec la colonisation des ligamentocytes. Enfin, l'objet mis en forme à partir du matériau devra pouvoir être stérilisé de façon à éviter tous

Ou module d'élasticité longitudinale. 


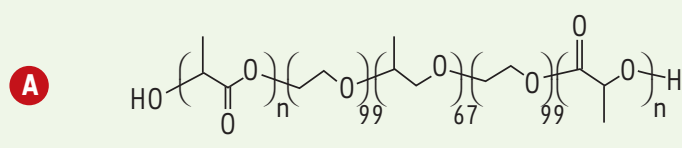

B

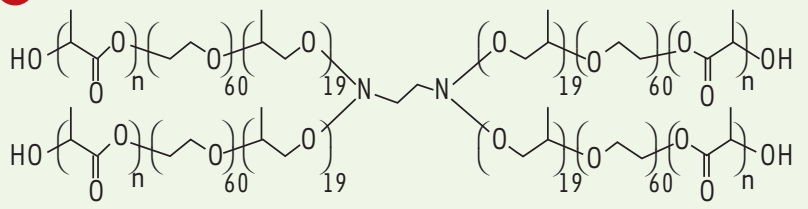

Figure 2. Structures des copolymères triblocs. A. PLA-Pluronic ${ }^{\odot}-\mathrm{PLA}$. B. PLATetronic $^{\odot}$-PLA. PLA : acide polylactique.

risques d'infection après l'implantation. Tous ces paramètres doivent être pris en compte dans la conception d'un biomatériau !

\section{Structure des copolymères : propriétés mécaniques}

L'étape fondamentale de cette recherche est l'obtention d'une structure chimique qui réponde au cahier des charges, tout particulièrement en ce qui concerne ses propriétés mécaniques. Pour des applications temporaires comme les dispositifs médicaux implantables [7], les systèmes de libération de principes actifs [8, 9] ou les matériaux pour l'ingénierie tissulaire $[10,11]$, les polyesters aliphatiques dérivés du lactide (LA), du glycolide (GA), ou de l'E-caprolactone $(C L)$, sont les plus utilisés car agréés par les agences de santé américaines et européennes. Mais, malgré leur excellente biocompatibilité, ces polymères ne peuvent posséder toutes les propriétés optimales pour de nombreuses applications [12-14]. II convient donc de modifier ces structures. La méthode la plus classique est la copolymérisation qui permet d'obtenir des composés contenant au moins deux motifs différents. Selon l'arrangement des motifs dans la chaîne, des copolymères statistiques ou séquencés (blocs) sont obtenus. Ces derniers sont, en règle générale, plus intéressants car ils permettent de cumuler les propriétés des deux homopolymères. Notre objectif était donc de développer des thermoplastiques élastomères ${ }^{2}$ (TPE) qui associent un monomère apportant des propriétés de rigidité et un monomère apportant des segments souples conférant l'élasticité, l'ensemble devant rester biocompatible et biodégradable. Le DMI (prothèse ligamentaire) jouera le rôle du tissu déficient le temps que celui-ci se reconstitue. Il disparaîtra ensuite de l'organisme par dégradation hydrolytique. Malheureusement, les polymères cités précédemment sont très rigides et très hydrophobes et, pour des applications comme biomatériau thermoplastique, il convient de moduler leurs caractéristiques mécaniques et l'hydrophilie. L'hydrophilie est couramment obtenue

${ }^{2}$ Les polymères thermoplastiques sont des matériaux macromoléculaires dont la caractéristique principale est leur possibilité de transformation réversible solide/liquide par apport de chaleur. Les élastomères ont des propriétés élastiques importantes. les thermoplastiques élastomères allient les deux propriétés. avec les copolymères PLA- $b$-PEG (PLA : acide polylactique; PEG : polyéthylène glycol) qui comportent une ou deux séquences de PLA (qui est hydrophobe) et une séquence de PEG, biocompatible et peu coûteux, qui confère l'hydrophilie au biomatériau. Bien que non intrinsèquement dégradable, le PEG est totalement éliminé de l'organisme par filtration rénale, après dégradation des unités PLA, pourvu que sa masse molaire ne soit pas trop élevée $\left(<20-30000 \mathrm{~g} \cdot \mathrm{mol}^{-1}\right)$. Les copolymères PLA- $b$-PEG présentent cependant de trop faibles caractéristiques mécaniques, en particulier au niveau du module d'young (5 à $70 \mathrm{MPa}$ pour le copolymère, contre 50 à $150 \mathrm{MPa}$ pour le LCA et 1,5-2 GPa pour le $P L A_{100}$ seul) et de la contrainte à la rupture (1 à $10 \mathrm{MPa}$ pour le copolymère, 16-36 MPa pour le LCA, et 12-20 MPa pour le PLA ${ }_{100}$ [15-17]).

Nous nous sommes intéressés à de nouveaux copolymères amphiphiles. Nous avons en particulier remplacé le PEG par le Pluronic $\mathrm{F} 127^{\odot}$ ou le Tetronic $1107^{\circ}$ (Figure 2) des polymères commerciaux hydrophiles agréés par les agences de santé. Les masses molaires moyennes en nombre du Pluronic ${ }^{\odot} \mathrm{F} 127$ et du Tetro$\mathrm{nic}^{\odot} 1107$ sont respectivement de $12600 \mathrm{~g} \cdot \mathrm{mol}^{-1}$ et $15000 \mathrm{~g} \cdot \mathrm{mol}^{-1}$, ce qui permet, comme pour le P\&G, leur élimination par filtration rénale. Des copolymères triblocs PLA-Pluronic ${ }^{\odot}$-PLA et PLA-Tetronic ${ }^{\odot}$-PLA ont été préparés et étudiés comme nouveaux thermoplastiques élastomères biodégradables [18]. Les propriétés mécaniques de ces copolymères ont été examinées en fonction de la longueur des segments PLA et de la nature du motif central hydrophile (Pluronic $^{\odot}$ et Tetronic $\left.^{(}\right)$. Le PLA est ici un PLA ${ }_{94}$ (c'est-à-dire qu'il est composé de $94 \%$ d'unités L-lactique et de $6 \%$ d'unités D-lactique). Ce stéréocopolymère particulier a été choisi car il représente un bon compromis entre propriétés mécaniques et vitesse de dégradation, toutes deux bien adaptées [19-22]. Il est en particulier non cristallin ce qui accroît, de manière notable, sa vitesse de dégradation par rapport à celle du $\mathrm{PLA}_{100}$. Les copolymères ont été préparés par polymérisation par ouverture de cycle du lactide amorcée par les extrémités alcool des Pluronic ${ }^{\odot}$ et Tetronic $^{\odot}$, en utilisant l'octanoate d'étain comme catalyseur, selon un procédé bien connu de la littérature.

Les principales propriétés mécaniques des copolymères obtenus sont présentées dans le Tableau / qui montre que les valeurs du module d'young observées avec ces copolymères sont plus élevées que celles des copolymères à base de PLA et de PEG. Les valeurs du module d'young et de l'allongement à la rupture sont même plus élevées que pour le LCA, ce qui présente certains avantages. En particulier, un allongement à la rupture 


\begin{tabular}{|c|c|c|c|c|c|c|c|}
\hline & & $\begin{array}{l}\text { Masse } \\
\text { molaire } \\
\text { (g/mole) } \\
\text { en RMN }\end{array}$ & $\begin{array}{l}\text { Module } \\
\text { d'Young } \\
\text { (MPa) }\end{array}$ & $\begin{array}{l}\text { Contrainte } \\
\text { à la rupture } \\
(\mathrm{MPa})\end{array}$ & $\begin{array}{c}\text { Allongement } \\
\text { à la rupture } \\
(\%)\end{array}$ & $\begin{array}{l}\text { Prise d'eau } \\
\text { initiale (\% } \\
\text { en masse) }\end{array}$ & $\begin{array}{c}\text { Dégradation à } \\
7 \text { semaines (\% masse } \\
\text { molaire initiale } \\
\text { restant) }\end{array}$ \\
\hline LCA & & - & $50-150$ & $16-36$ & $19-36$ & & \\
\hline PLA & & 200000 & 800 & 40 & 30 & & \\
\hline PLA/PEG* & & & $5-70$ & $1-10$ & & & \\
\hline PLA/Pluronic & $94 P 100$ & 120000 & 270 & 12 & 770 & 15 & 55 \\
\hline PLA/Pluronic & $94 P 200$ & 220000 & 550 & 18 & 600 & 7 & 85 \\
\hline PLA/Tetronic & $94 T 100$ & 115000 & 230 & 11 & 830 & 9 & 55 \\
\hline PLA/Tetronic & $94 T 200$ & 240000 & 450 & 20 & 760 & 6 & 80 \\
\hline
\end{tabular}

Tableau I. Propriétés mécaniques et dégradation in vitro des différents copolymères à base de PLA, Tetronic et Pluronic. *Pour les faibles masses molaires, les valeurs des divers paramètres sont non mesurables. LCA : ligament croisé antérieur du genou ; PLA : polyesters aliphatiques dérivés du lactide ; PEG : polyéthylène glycol ; RMN : résonance magnétique nucléaire.

élevé est intéressant pour la solidité du ligament artificiel lorsque celui-ci est soumis à un étirement. II est d'autre part connu que la stérilisation ultérieure peut conduire à des coupures de chaînes qui engendrent des pertes de propriétés mécaniques et une augmentation de la vitesse de dégradation [23].

L'influence de la masse molaire des blocs PLA sur les propriétés mécaniques et sur l'hydrophilie, mesurée par la prise d'eau initiale est également examinée. Comme attendu, les copolymères dont les chaînes de PLA sont les plus longues présentent les meilleures propriétés mécaniques et les absorptions d'eau les plus faibles, ils se dégraderont donc le moins rapidement. En effet, bien qu'ils subissent une dégradation, ils conservent plus de $80 \%$ de leur masse molaire après sept semaines. Compte tenu des résultats relatifs aux propriétés mécaniques et à la vitesse de dégradation, les copolymères PLA-Pluronic $^{\odot}$ et PLA-Tetronic ${ }^{\odot}$ de fortes masses molaires ont ainsi été retenus.

\section{Stérilisation}

La stérilisation du biomatériau est une étape obligatoire pour des applications médicales. Cette étape primordiale est cependant connue pour dégrader les chaînes polymères, surtout les polymères dégradables, et donc, en premier lieu, les propriétés mécaniques et la vitesse de dégradation du biomatériau. Les copolymères que nous avons retenus ont été stérilisés par rayonnements gamma (entre 31,5 et 39 kGy [kilo gray]) ou bêta (entre 25 et 27 kGy) sous atmosphère normale. Les variations du module d'young, de la contrainte et de l'allongement à la rupture des copolymères 94 P200 et 94 T200 avant et après stérilisation sont présentées dans le Tableau II.

Les variations observées peuvent être importantes et relèvent de deux phénomènes différents: les coupures de chaînes et la réticulation. On note en particulier, après stérilisation $\gamma$, la forte diminution de l'allongement à la rupture et l'augmentation du module d'young due à une réticulation du système. La forte diminution de l'allongement à la rupture est en particulier très préjudiciable pour I'application ciblée. La stérilisation $\beta$ qui impacte moins les propriétés sera donc préférée.

\section{Dégradation hydrolytique des copolymères}

Avant d'être éliminé de l'organisme, le biomatériau doit conserver les propriétés mécaniques du genou tout en permettant une recolonisation et une maturation cellulaire suffisantes pour permettre la création d'un néoligament mature résistant. Une étude de la dégradation des copolymères $94 \mathrm{P} 200$ et $94 \mathrm{P} 100$ a été réalisée in vitro en utilisant un tampon phosphate à $\mathrm{pH} 7,4$ à $37^{\circ} \mathrm{C}$. La variation des masses molaires des copolymères a été étudiée par comparaison avec celles qu'ils présentaient avant dégradation. On observe ainsi que plus les chaînes de PLA sont longues, plus la dégradation est lente. En effet, pour les copolymères 94P100 et 94T100, la masse molaire diminue de $40 \%$ contre $22 \%$ pour le 94 P200 et le 94T200 qui présentent de longues chaînes de PLA. Pour les copolymères $94 \mathrm{P} 100$ et $94 \mathrm{~T} 100$, les propriétés mécaniques ne peuvent plus être mesurées après sept semaines de dégradation car ils sont trop dégradés et les objets à analyser sont devenus cassants. Cette perte des propriétés mécaniques dans ce délai de sept semaines rend ces copolymères inadaptés à l'application. Pour les $94 \mathrm{P} 200$ et $94 \mathrm{~T} 200$, les principales conséquences mécaniques résident dans l'allongement à la rupture qui passe en deux semaines de $600 \%$ à $100 \%$ pour le $94 \mathrm{P} 200$, et de $760 \%$ à $20 \%$ pour le $94 \mathrm{~T} 200$. Notons qu'il ne varie que très peu pour l'homopolymère $\mathrm{PLA}_{94}$, preuve de l'importance de l'hydrophilie sur la vitesse de dégradation [18]. 


\begin{tabular}{|c|c|c|c|}
\hline & Non stérilisé & Stérilisé $\beta$ & Stérilisé $\gamma$ \\
\hline \multicolumn{4}{|c|}{ Module d'Young (MPa) } \\
\hline $94 P 200$ & $555 \pm 50$ & $452 \pm 100$ & $867 \pm 27$ \\
\hline $94 T 200$ & $455 \pm 24,5$ & $439 \pm 48,1$ & $656 \pm 123$ \\
\hline$L C A$ & $50-150$ & & \\
\hline \multicolumn{4}{|c|}{ Contrainte à la rupture $(\mathrm{MPa})$} \\
\hline $94 P 200$ & $18,0 \pm 1,6$ & $13 \pm 2,0$ & $15,4 \pm 5,8$ \\
\hline $94 T 200$ & $20,0 \pm 1,5$ & $19,8 \pm 3,8$ & $16,9 \pm 5,5$ \\
\hline$L C A$ & $16-36$ & & \\
\hline \multicolumn{4}{|c|}{ Allongement à la rupture (\%) } \\
\hline $94 P 200$ & $600 \pm 40$ & $545 \pm 200$ & $10,3 \pm 1,9$ \\
\hline $94 T 200$ & $760 \pm 55$ & $965 \pm 150$ & $12,3 \pm 6,0$ \\
\hline$L C A$ & $19-36$ & & \\
\hline
\end{tabular}

Tableau II. Variation des propriétés mécaniques en fonction du mode de stérilisation. LCA : ligament croisé antérieur du genou.

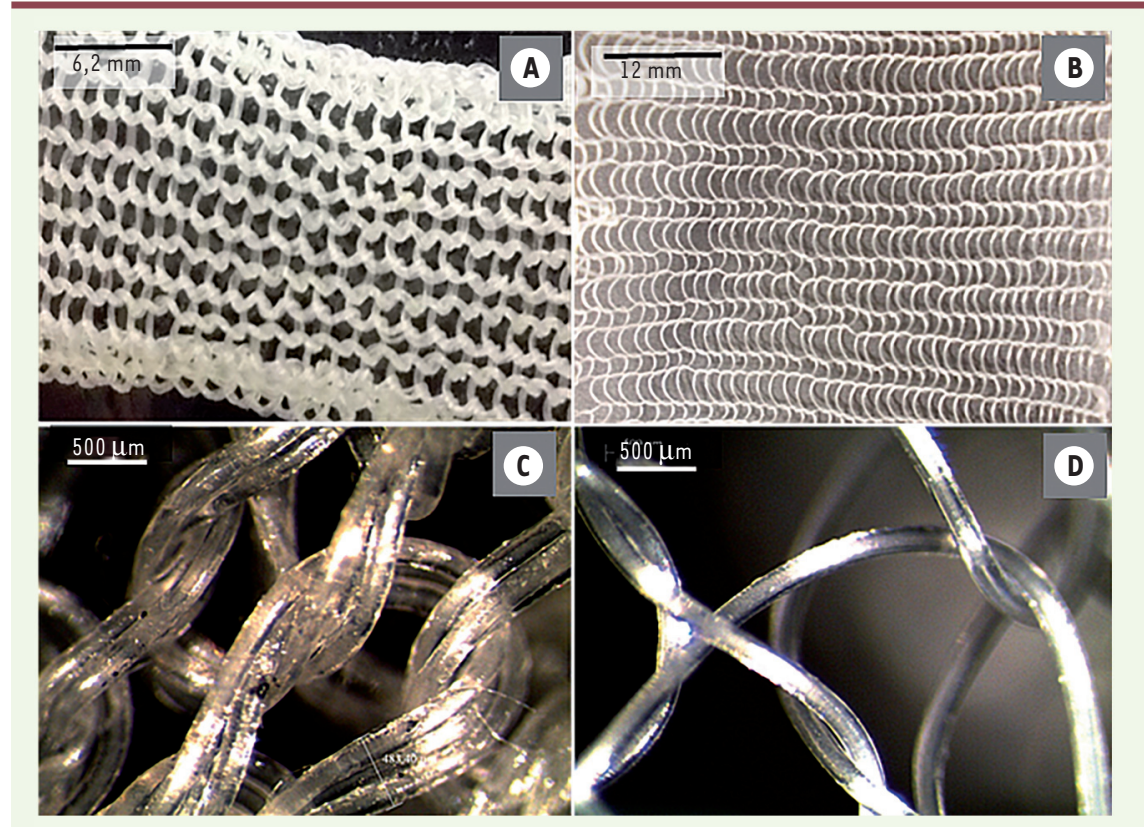

Figure 3. Illustrations en microscopie optique des tricots obtenus à partir de multifilaments $(A, C)$ et à partir de monofilaments $(B, D)$ de copolymère dégradable.

sections plus ou moins grandes (de 0,1 à $1 \mathrm{~mm}$ ). D'une manière générale, cette opération entraîne des coupures de chaînes (diminution de masse molaire de l'ordre de 10 à $20 \%$ ) et une augmentation de la cristallinité du matériau qui induit une variation des propriétés mécaniques, notamment du module d'young (de environ $500 \mathrm{MPa}$ à environ $1300 \mathrm{MPa}$ ) et de la résistance à la rupture (d'environ $20 \mathrm{MPa}$ à environ $120 \mathrm{MPa}$ ).

Les microfilaments sont ensuite utilisés seuls (monofilaments) ou associés par 10 (multifilaments). Les mono ou

\section{Mise en forme}

Les principales propriétés mécaniques et de dégradation des copolymères étant établies, diverses étapes de mise en forme sont nécessaires afin d'obtenir un objet fini. Pour servir de support de colonisation tissulaire, le polymère a été mis en forme de façon à obtenir une structure cylindrique et poreuse de type tresse ou tricot (Figure 3). Des microfilaments ont d'abord été réalisés par passage du copolymère fondu dans une filière suivi d'un étirage afin d'obtenir des multifilaments sont tricotés à l'aide d'une machine à tricoter semi-automatique pour donner des tricots (Figure 3) dont la taille moyenne des mailles varie entre 0,65 et $1,3 \mathrm{~mm}$. Comme le fil nécessaire à la confection du tissu est plus épais dans le tricot multifilament, il en résulte un textile final beaucoup plus dense, intéressant notamment pour ses propriétés élastiques et sa forte résistance à la rupture. À partir de ce tricot multifilament, un renfort tubulaire en trois dimensions a été 


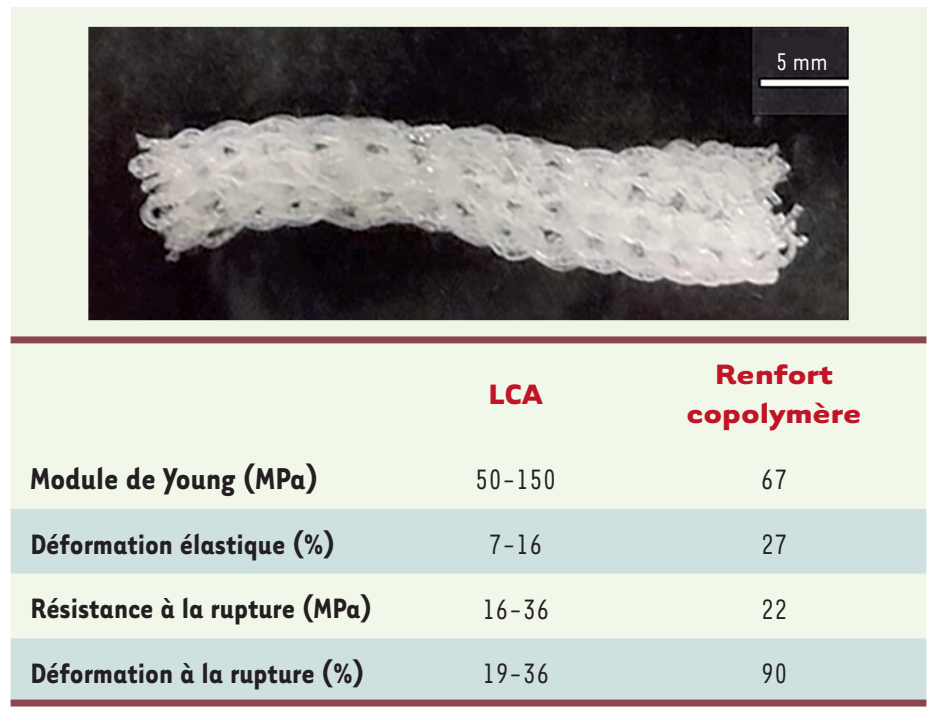

Figure 4. Image du renfort total ligamentaire en copolymère dégradable tricoté et propriétés mécaniques de celui-ci. LCA : ligament croisé antérieur du genou.

confectionné afin d'obtenir une structure mimant celle d'un ligament croisé antérieur natif (Figure 4). Avec un module d'Young de $67 \mathrm{Mpa}$, une déformation élastique de $27 \%$ et une contrainte à la rupture de 22 $\mathrm{Mpa}$, les valeurs caractéristiques du renfort tubulaire se rapprochent de celles du ligament croisé antérieur natif (Figue 4) [5].

\section{Cytocompatibilité}

Si les constituants des copolymères (PLA, Pluronic ${ }^{\oplus}$ et Tetronic ${ }^{\oplus}$ ) sont effectivement cytocompatibles, il convient cependant de vérifier celle des copolymères synthétisés. Dans ce but la viabilité et la prolifération de cellules C3 MSC (une lignée de cellules souches mésenchymateuses) ont été étudiées par culture à la surface de disques de copolymères durant sept jours [19]. Aucune différence de viabilité des cellules n'a été observée sur les copolymères par rapport aux homopolymères, ou au contrôle sur TCPS (tissue culture polystyrene). La cytocompatibilité a été confirmée par un test Live and Dead ${ }^{\circledR}$ présenté dans la Figure 5 réalisé avec le 94P200. Les cellules prolifèrent et couvrent entièrement la surface du copolymère (Figure $5 \mathrm{~A}$ ) et pratiquement aucune cellule morte n'est détectée (Figure 5B). Ces résultats confirment non seulement la cytocompatibilité des copolymères, mais ils montrent également que la structure est compatible avec la colonisation cellulaire, ce qui est très intéressant pour les applications biologiques considérées.

\section{Conclusion}

Dans le but de développer de nouveaux biomatériaux dégradables répondant aux principaux critères du remplacement du ligament croisé antérieur du genou, des copolymères blocs PLA-Pluronic ${ }^{\circledR} \mathrm{F} 127$ et PLA-Tetronic ${ }^{\circledR} 1107$ ont été développés et testés. L'importance de la structure des copolymères sur les propriétés, notamment les propriétés mécaniques et la vitesse de dégradation, qui sont primor-

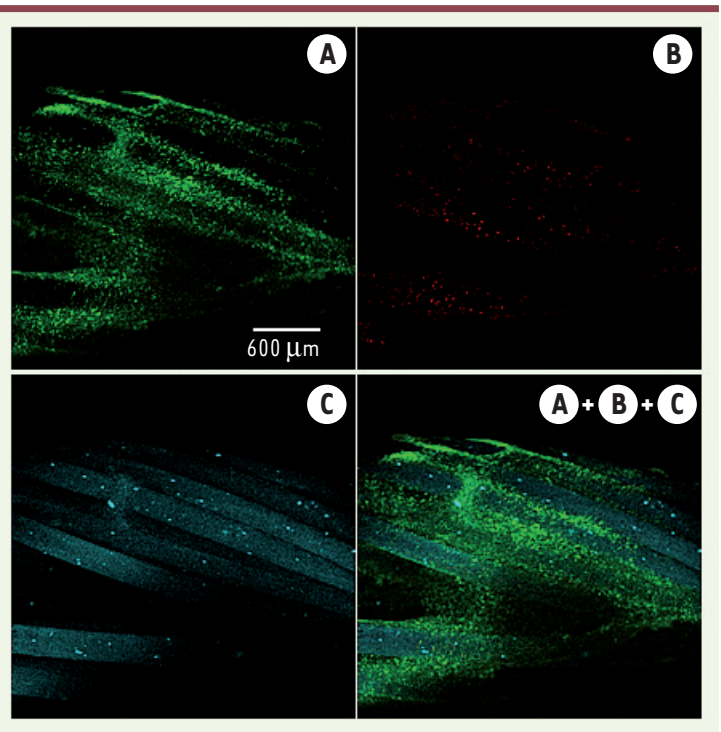

Figure 5. Observation par microscopie confocale à fluorescence de cellules $C 3$ après 10 jours de culture sur une matrice textile et marquage Live/Dead ${ }^{\circledR}$. Les cellules vivantes sont en vert $(A)$, les cellules mortes en rouge (B), l'auto-fluorescence du polymère est présentée en bleu (C).

diales pour cette application, a été mise en évidence et a guidé le choix de ces deux copolymères. La sélection du PLA pour sa biodégradabilité modulable, et du Pluronic ${ }^{\odot}$ ou du Tetronic ${ }^{\odot}$ pour l'apport de l'hydrophilie au système, à la place du classique PEG, a pu être justifiée. L'effet sur les propriétés mécaniques comme sur la vitesse de dégradation de la longueur des chaînons hydrophobes de PLA a été mis en évidence. Cette longueur a pu être adaptée en tenant compte des étapes nécessaires de stérilisation et de mise en forme, qui sont connues pour modifier ces propriétés. Le mode de stérilisation choisi a été une stérilisation par rayons $\beta$ qui impacte le moins possible les propriétés mécaniques. Un renfort tubulaire en trois dimensions a enfin été confectionné. II répond aux cahiers des charges d'un LCA artificiel, en termes de biocompatibilité, de propriétés mécaniques, de vitesse de dégradation et de cytocompatibilité.

D’une manière générale, les biomatériaux utilisés pour concevoir des dispositifs médicaux implantables doivent être développés sous la forme d'objet ou de textile, stérilisés et assurer une bonne biocompatibilité. La conception de tels produits fait donc appel aux compétences transversales de chimistes, de spécialistes des synthèses des polymères et des propriétés des matériaux, de biologistes et de médecins. C'est donc la réunion de ces compétences qui est essentielle dans les stratégies de recherche dans le domaine des dispositifs médicaux implantables. $\diamond$ 


\section{SUMMARY}

Biodegradable synthetic polymers for the design of implantable medical devices: the ligamentoplasty case

The sector of implantable medical devices is a growing sector of health products especially dynamic in the field of research. To improve the management of patients and to meet clinical requirements, researchers are developing new types of medical devices. They use different families of biomaterials presenting various chemical and physical characteristics in order for providing clinicians with health products optimized for biomedical applications. In this article, we aim to show how, starting from a family of biomaterials (degradable polymers), it is possible to design an implantable medical device for the therapeutic management of the failure of anterior cruciate ligament. The main steps leading to the design of a total ligament reinforcement are detailed. They range from the synthesis and characterization of degradable polymer to the shaping of the knitted implant, through the assessment of the study of the impact of sterilization on mechanical properties and checking cytocompatibility. $\diamond$

\section{LIENS D'INTÉRÊT}

Les auteurs n'ont pas transmis de déclaration de liens d'intérêt.

\section{RéFÉRENCES}

1. Mac Chesney B. Global medical device market size, growth, and trends by therapeutic area (20092017), $1^{\text {st }}$ ed. DeciBio, 2014 : 102 p.

2. De Smedt M. Les prothèses du ligament croisé anterieur : analyse d'un échec. Acta Orthopaedica Belgica $1998 ; 64: 422-33$.

3. Guidoin MF, Marois Y, Bejui J, et al. Analysis of retrieved polymer fiber based replacements for the ACL. Biomaterials $2000 ; 21: 2461-74$.

4. Goodship AE, Cooke PH. The influence of a biomechanically matched composite cruciate ligament prosthesis on subsequent joint function and degenerative change: an experimental-study.J Biomech $1987 ; 20: 810$.

5. Vieira AC, Guedes RM, Marques AT. Development of ligament tissue biodegradable devices: a review. J Biomech 2009; $42: 2421-30$.

6. Marumo K, Saito M, Yamagishi T, Fujii K. The ligamentization process in human anterior cruciate ligament reconstruction with autogenous patellar and hamstring tendons: a biochemical study. Am J Sport Med $2005 ; 33$ : 1166-73.

7. Lyu SP, Untereker D. Degradability of polymers for implantable biomedical devices. Int J Mol Sci $2009 ; 10: 4033-65$.

8. Biondi M, Ungaro F, Quaglia F, Netti PA. Controlled drug delivery in tissue engineering. Adv Drug Deliv Rev $2008 ; 60: 229-42$.
9. Kretlow JD, Klouda L, Mikos AG. Injectable matrices and scaffolds for drug delivery in tissue engineering. Adv Drug Deliv Rev 2007 ; 59 : 263-73.

10. Dhandayuthapani B, Yoshida Y, Maekawa T, Kumar DS. Polymeric scaffolds in tissue engineering application: a review. Int J Polymer Science 2011. Article ID 290602, 19 pages, doi:10.1155/2011/290602.

11. Langer R, Tirrell DA. Designing materials for biology and medicine. Nature $2004 ; 428: 487-92$

12. Folliguet TA, Rucker-Martin C, Pavoine C, et al. Adult cardiac myocytes survive and remain excitable during long-term culture on synthetic supports. J Thorac Cardiovasc Surg 2001; $121: 510-9$.

13. Surrao DC, Fan JCy, Waldman SD, Amsden BG. A crimp-like microarchitecture improves tissue production in fibrous ligament scaffolds in response to mechanical stimuli. Acta Biomaterialia $2012 ; 8$ : 3704-13.

14. Surrao DC, Waldman SD, Amsden BG. Biomimetic poly (lactide) based fibrous scaffolds for ligament tissue engineering. Acta Biomaterialia 2012 ; 8 : 3997-4006.

15. Fernandez J, Etxeberria A, Ugartemendia JM, et al. Effects of chain microstructures on mechanical behavior and aging of a poly (L-lactide-coepsilon-caprolactone) biomedical thermoplastic-elastomer.J Mech Behav Biomed Mater $2012 ; 12$ : 29-38.

16. Lipik VT, Kong JF, Chattopadhyay S, et al. Thermoplastic biodegradable elastomers based on epsilon-caprolactone and L-lactide block co-polymers: a new synthetic approach. Acta Biomaterialia $2010 ; 6$ : 426170.

17. Zhang Z, Grijpma DW, Feijen J. Thermoplastic elastomers based on poly(lactide)-poly (trimethylene carbonate-co-caprolactone)poly(lactide) triblock copolymers and their stereocomplexes. J Control Release 2006 ; 116 : ع29-દ31.

18. Leroy A, Pinese C, Bony C, et al. Investigation on the properties of linear PLA-poloxamer and star PLA-poloxamine copolymers for temporary biomedical applications. Mater Sci Eng C Mater Biol Appl 2013 ; 33 : 4133-9.

19. Leroy A, Nottelet B, Bony C, et al. PLA-poloxamer/poloxamine copolymers for ligament tissue engineering: sound macromolecular design for degradable scaffolds and MSC differentiation. Biomater Sci $2015 ; 3: 617-26$.

20. Li SM, Garreau H, Vert M. Structure property relationships in the case of the degradation of massive aliphatic poly-(alpha-hydroxy acids) in aqueousmedia. 1. Poly(DI-lactic acid). J Mater Sci Mater Med 1990 ; 1 : 123-30.

21. Vert M, Li SM, Garreau H. Attempts to map the structure and degradation characteristics of aliphatic polyesters derived from lactic and glycolic acids. J Biomater Sci Polym Ed $1994 ; 6: 639-49$.

22. Vert M, Li SM, Spenlehauer G, Guerin P. Bioresorbability and biocompatibility of aliphatic polyesters. J Mater Sci Mater Med 1992 ; 3 : 432-46.

23. Babanalbandi A, Hill DJT, Hunter DS, Kettle L. Thermal stability of poly (lactic acid) before and after gamma-radiolysis. Polymer International $1999 ; 48$ : $980-4$.

\section{LA FONDATION PREMUP : UN OPÉRATEUR DE TERRAIN EN PÉRINATALITÉ RECONNU POUR SON EXCELLENCE ET SON INTERDISCIPLINARITÉ}

La Fondation de coopération scientifique PremUp, unique en Europe, intervient sur la prévention du handicap à la naissance, par la protection de la santé de la femme enceinte et du nouveau-né.

\section{TIRÉS À PART}

X. Garric

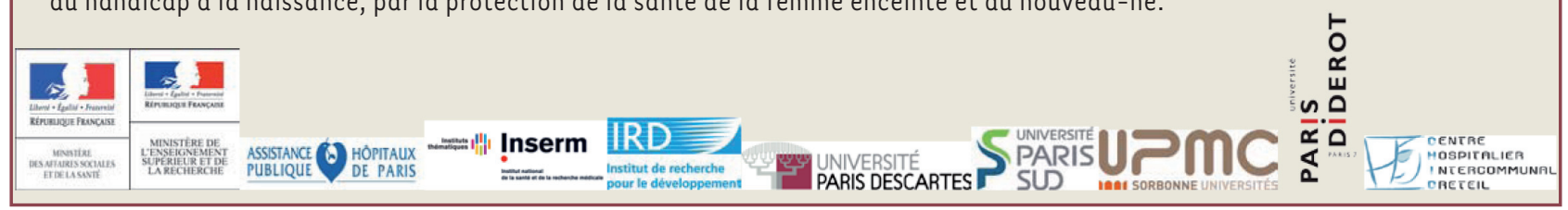

